\title{
The Diagnosis of Spontaneous Coronary Artery Dissection by Optical Coherence Tomography
}

\author{
Takahiro Kanda, Kei Tawarahara, Gaku Matsukura, Masayoshi Matsunari, Rumi Takabayashi, \\ Jun Tamura, Mariko Ozeki and Hiroshi Ukigai
}

\begin{abstract}
:
Spontaneous coronary artery dissection (SCAD) is rare, but it frequently presents as acute myocardial infarction. It is frequently fatal and most cases are diagnosed at autopsy. We herein present the case of a 65year-old woman with ST-elevation and myocardial infarction due to SCAD. Optical coherence tomography (OCT) helped us to confirm the diagnosis. The information on the intravascular morphology provided by OCT imaging is much more detailed in comparison to that provided by coronary angiography (CAG) and intravascular ultrasound (IVUS).
\end{abstract}

Key words: spontaneous coronary artery dissection, optical coherence tomography

(Intern Med 57: 523-526, 2018)

(DOI: 10.2169/internalmedicine.8862-17)

\section{Introduction}

Spontaneous coronary artery dissection (SCAD), an infrequent cause of acute coronary syndrome, is often fatal and is the leading cause of pregnancy-related myocardial infarction during the postpartum period. Moreover, its diagnosis might be underestimated and its natural history and etiology have yet to be elucidated. Here, we report a case of SCAD involving the left anterior descending artery in a 65-year-old woman that presented with acute coronary syndrome. Using optical coherence tomography (OCT), SCAD and intramural hematoma were diagnosed. Furthermore, because TIMI3 blood flow was reserved on coronary angiography (CAG), we selected conservative medical therapy. Four months later, follow-up CAG and OCT revealed a completely repaired coronary artery narrowing and wall structure.

\section{Case Report}

A 65-year-old woman with essential hypertension, dyslipidemia, and a family history as coronary risk factors visited a local physician's office with chest discomfort and throat pain that persisted for 3 hours. A 12-lead electrocardiogram showed an ST elevation in the V2-4 leads; she was therefore transferred to our hospital by ambulance. Her symptoms had disappeared by the time she arrived. A physical examination revealed a regular pulse of 114 beats $/ \mathrm{min}$ and a blood pressure of 172/94 mmHg. A 12-lead electrocardiogram showed terminal $\mathrm{T}$ inversion in the V3-4 leads. The echocardiogram demonstrated akinesis of the apical anteroseptal wall, and the laboratory data showed elevated creatine phosphokinase (960 IU/L) and troponin I levels (13.9 ng/mL).

Emergent CAG revealed diffuse narrowing in the distal left anterior descending artery (LAD-distal) and focal stenosis at the first diagonal branch bifurcation lesion (LAD-D1 bifurcation; Fig. 1A). No angiographic findings of dissection were recognized and the TIMI3 flow was reserved. We first thought the LAD-distal or LAD-D1 bifurcation was the culprit lesion. To evaluate the intravascular morphology of these two lesions, we performed OCT.

At the LAD-distal lesion, OCT demonstrated diffuse lumen compromise and intramural hematoma between the intima and media but did not visualize the intimomedial flap and entry tear or find any vulnerable plaque, such as a thincap fibroatheroma (TCFA), erosion, or thrombi in the true lumen (Fig. 2). Moreover, OCT revealed a fibrous plaque at the LAD-D1 bifurcation lesion. We therefore thought that the acute coronary syndrome had been caused by SCAD and an intramural hematoma at the LAD-distal lesion. 
Because the TIMI3 blood flow was reserved in the left anterior descending artery, we selected conservative medical

A
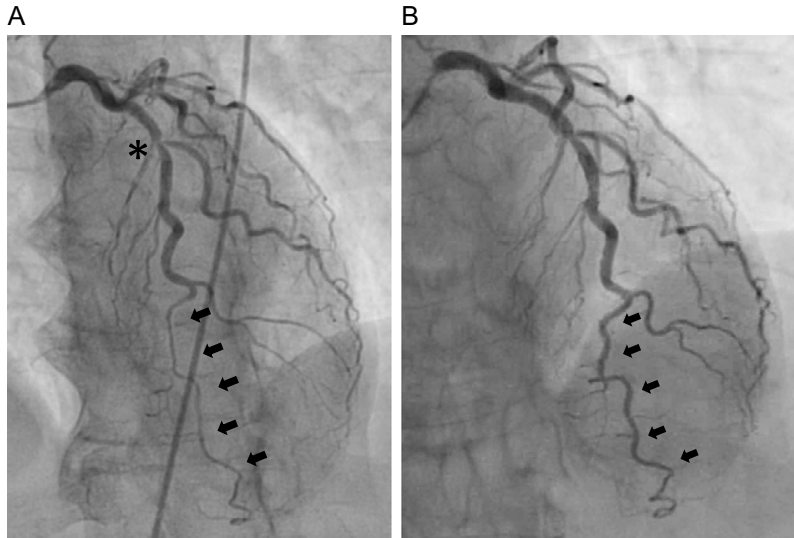

Figure 1. A: Coronary angiography $\left(\mathrm{LAO30}^{\circ} / \mathrm{CRA30}^{\circ}\right.$ view $)$ Emergent coronary angiography revealed diffuse narrowing in the distal left anterior descending artery (arrows) and focal stenosis at the first diagonal branch bifurcation site (*). B: Follow up coronary angiography $\left(\mathrm{LAO30} / \mathrm{CRA30}^{\circ}\right.$ view). Follow-up CAG revealed the late spontaneous resolution of the angiographic findings corresponding to SCAD. therapy. No complications were noted, and the patient was discharged from our hospital on day 15. Late gadolinium enhancement was observed in a small area of the apical segment on cardiac magnetic resonance imaging. At four months after the onset of symptoms, follow-up CAG and OCT revealed late spontaneous resolution of the angiography and OCT findings, corresponding to SCAD (Fig. 1B, 3).

\section{Discussion}

SCAD is relatively common among young to middle-aged women and it is often reported as a cause of acute myocardial infarction (AMI). Conventionally, SCAD is diagnosed when CAG confirms findings of dissection or a diffuse narrowing lesion. However, several cases show unclear findings; therefore, it is difficult to accurately diagnose this disease using only CAG as a diagnostic tool.

OCT is an excellent technique for assessing vessel wall properties, plaque characteristics, the presence of thrombi, and other conditions and is very useful for differentiating the culprit lesion of myocardial infarction and surmising events within the blood vessels. To date, several reports have
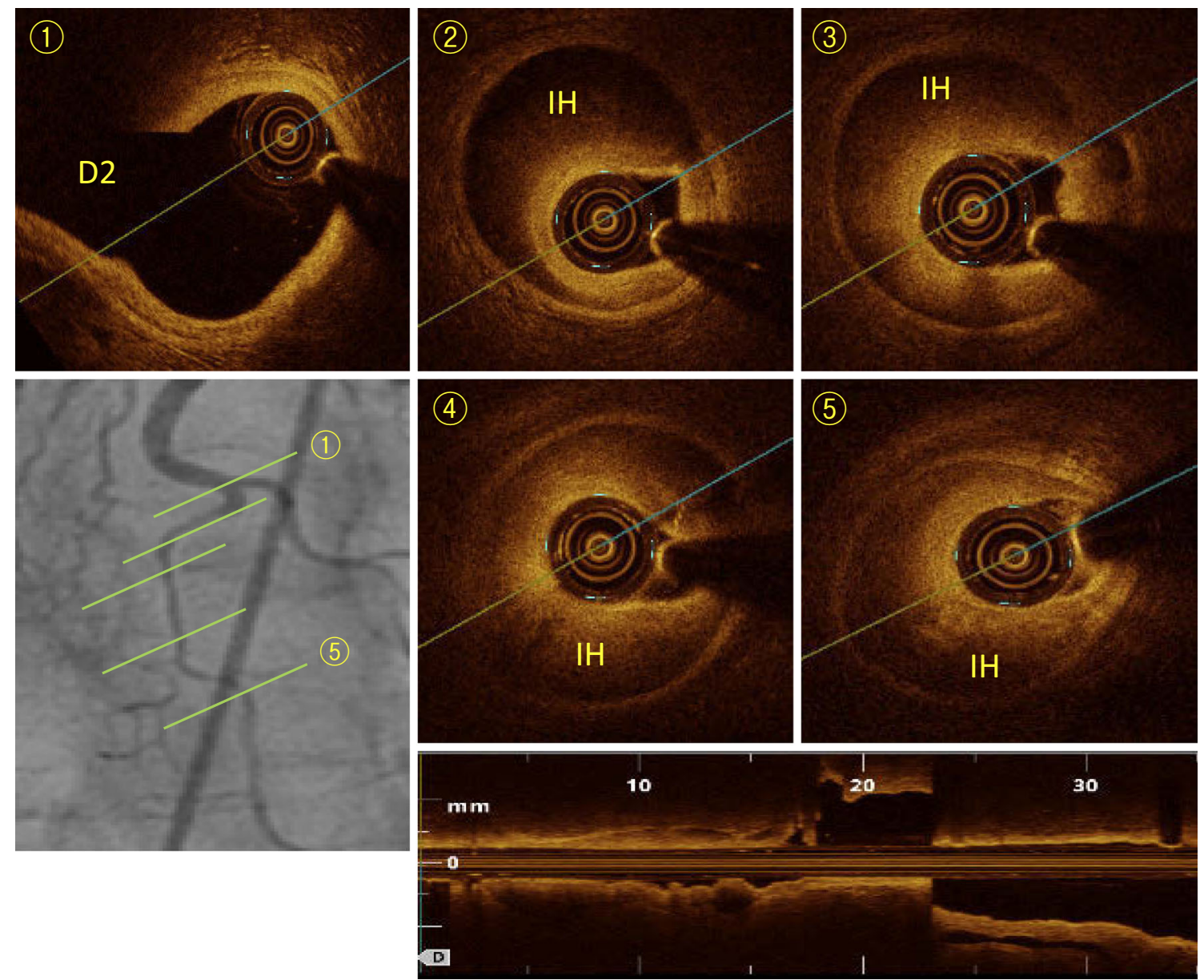

Figure 2. OCT in emergent CAG. (1)LAD-D2 bifurcation: Entry tear was not observed. (2)-(5)LADdistal diffuse coronary narrowing: The lumen compromise and the diffuse low density lesion that was suspected of being an intramural hematoma between the intima and media were observed. D2: second diagonal branch, IH: intramural hematoma 


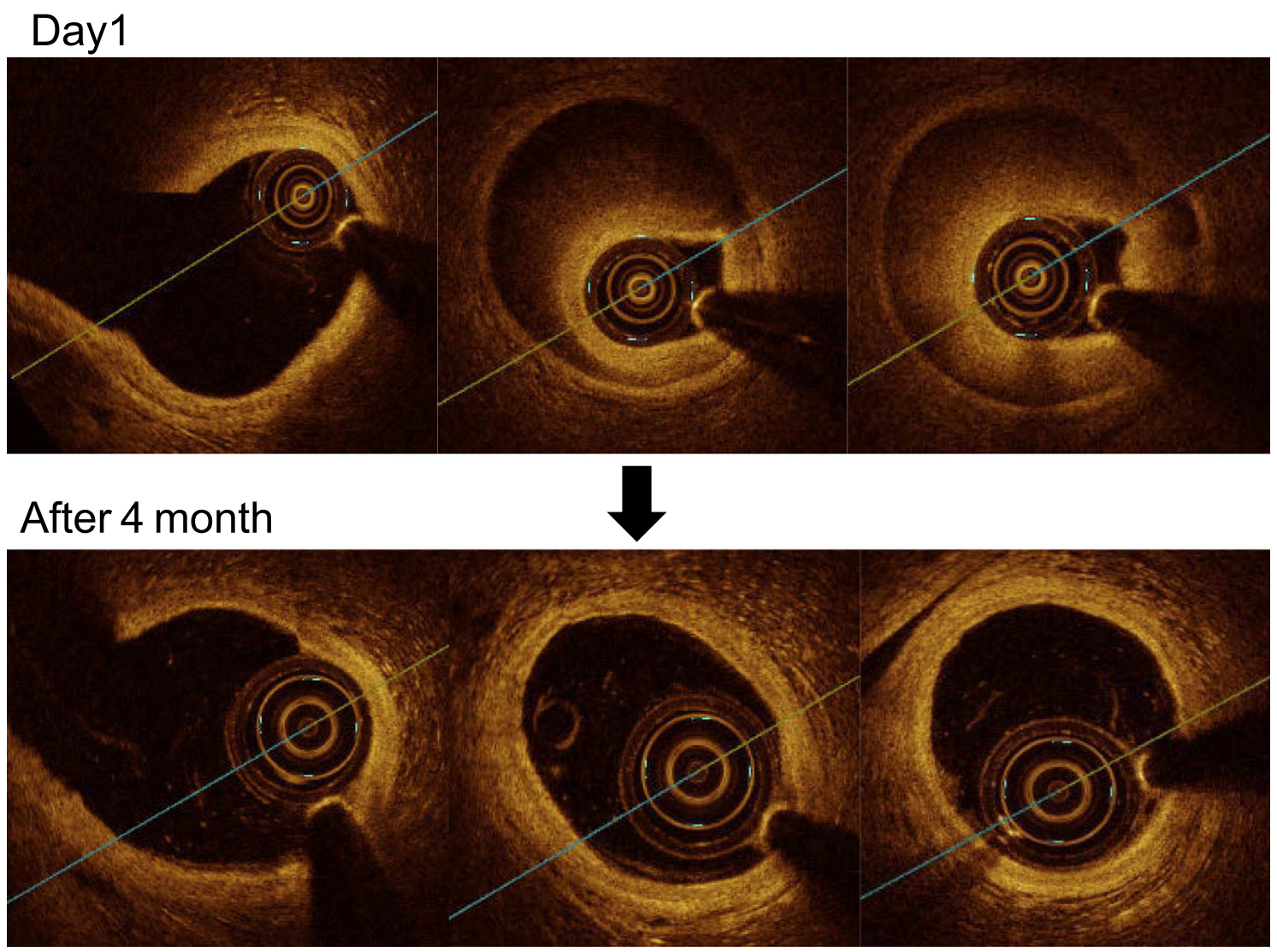

Figure 3. OCT at 4 months after the onset of symptoms. The LAD-distal lesion showed reparation with three intact arterial wall layers.

described the efficacy of OCT in diagnosing and subsequently formulating a treatment strategy for patients presenting with SCAD (1-4). The following aspects make OCT useful for diagnosing this disease: 1) lesions between the tunica intima and media can be accurately visualized; 2) the boundary between the true and false lumen is clearly and distinctly noted; 3) presence/absence of thrombus occluding the false lumen can be assessed; 4) the entire length of a dissection can be visualized in a long-axis image; and 5) the presence of a vulnerable plaque or thrombus can be easily confirmed. Among the imaging modalities that are used to observe the coronary arteries, intravascular ultrasound (IVUS) is often compared to OCT. It provides useful pictures of the underlying substrate, even in patients without antegrade coronary flow and in those with an occlusive intracoronary thrombus. Due to its ability to penetrate deeper into the vessel wall, IVUS can help to completely visualize large hematomas and penetrate red thrombi that cause major shadowing on OCT.

Based on the findings and the clinical course observed in the present case, the following examination factors were to considered to be indicators for selecting conservative treatment: 1) reserved thrombolysis in myocardial infarction (TIMI 2-3) blood flow; 2) the presence of a thrombus occluding the false lumen; 3) confirmation that there is no entry tear of the dissection; and 4) the absence of a thrombus in the true lumen. However, in cases in which only TIMI
0-1 blood flow was observed, CAG was found to provide poor imaging of the periphery of lesions, and even OCT failed to wash out the blood sufficiently, which implies that SCAD is difficult to diagnose in such situations. Thus, balloon dilatation or-in some cases-stent placement may be useful prior to using an intravascular imaging modality, and it is likely that SCAD may remain undiagnosed. In cases in which contrast medium pooling is confirmed by CAG, the use of OCT is inappropriate because of the risk of expanding the dissection cavity secondary to the injection of contrast medium. Thus, although OCT may not necessarily diagnose SCAD in all cases, this modality remains a very useful diagnostic technique in cases in which at least TIMI2-3 blood flow is reserved.

In our case, the intramural hematoma disappeared completely after 4 months, and the three-layer structure of normal vessels was observed to be reconstructed. Very few reports have described the efficacy of intracoronary imaging modalities in observing the spontaneous healing process of SCAD. Davlouros et al. (5) reported that OCT was used to confirm the spontaneous healing of SCAD within 4 months. Alfonso et al. (6) also reported the SCAD spontaneously disappeared in 7 of 13 patients during 6 years of follow-up. The exact mechanism underlying the spontaneous resolution of SCAD is unknown and has not been explained in the literature. It has been hypothesized that thrombus formation in the false lumen occludes the false lumen and causes fibrosis 
between the two dissected layers in cases with aortic dissection (7). It is presumed that the intramural hematoma found in the false lumen is gradually absorbed due to some unknown mechanism, and that finally, the inner membrane and media are reorganized with the restoration of the normal three-layer structure of blood vessels. Within this decade, OCT will elucidate the real healing process in SCAD.

The authors state that they have no Conflict of Interest (COI).

\section{References}

1. Alfonso F, Canales E, Aleong G. Spontaneous coronary artery dissection: diagnosis by optical coherence tomography. Eur Heart $\mathrm{J}$ 30: $385,2009$.

2. Combaret N, Souteyrand G, Amonchot A, et al. Contribution of guidance by optical coherence tomography (OCT) in rescue management of spontaneous coronary artery dissection. Eur Heart J Cardiovasc Imaging 14: 714, 2013.

3. Igleasias D, Salinas P, Jimenez-Valero S. Spontaneous coronary ar- tery dissection evaluated by optical coherence tomography. J Cardiovasc Med 12: 743-744, 2011.

4. Ishibashi K, Kitabata H, Akasaka T. Intracoronary optical coherence tomography assessment of spontaneous coronary artery dissection. Heart 95: 818, 2009.

5. Davlouros P, Xanthopoulou I, Tsigkas G, et al. Complete healing of spontaneous coronary artery dissection demonstrated by optical coherence tomography in a young postpartum female presenting with acute coronary syndorome. JACC Cardiovasc Interv 10: 8990, 2017.

6. Alfonso F, Paulo M, Lennie V, et al. Spontaneous coronary artery dissection: longterm follow-up of a large series of patients prospectively managed with a conservative therapeutic strategy. JACC Cardiovasc Interv 5: 1062-1070, 2012.

7. Kong $\mathrm{CH}$, Lin XY, Caleb MG, et al. Resolution of ascending aortic dissection in a Stanford type A patient. Ann Thorac Surg 96: 1066-1067, 2013.

The Internal Medicine is an Open Access article distributed under the Creative Commons Attribution-NonCommercial-NoDerivatives 4.0 International License. To view the details of this license, please visit (https://creativecommons.org/licenses/ by-nc-nd/4.0/).

(C) 2018 The Japanese Society of Internal Medicine Intern Med 57: 523-526, 2018 